\title{
A Fuzzy Decision Making Trial and Evaluation Laboratory Analysis of SCM System Implementation
}

\author{
Ta-Ping Lu ${ }^{1, *}$, Pei-Luen Patrick Rau ${ }^{2}$, Tang-Zuo Liou ${ }^{3}$ and Yi-Hsin Yang ${ }^{4}$ \\ ${ }^{1}$ Department of Industrial Engineering and Management, National Taipei University of Technology 1, Sec. 3, Chung-Hsiao E. Rd., \\ Taipei, Taiwan, R.O.C \\ ${ }^{2}$ Department of Industrial Engineering, Tsinghua University, Beijing 100084, China \\ ${ }^{3}$ Graduate Institute of Industrial Engineering and Management, National Taipei University of Technology 1, Sec. 3, Chung-Hsiao E. \\ Rd., Taipei, Taiwan, R.O.C \\ ${ }^{4}$ Graduate Institute of Industrial Engineering and Management, National Taipei University of Technology 1, Sec. 3, Chung-Hsiao E. \\ Rd., Taipei, Taiwan, R.O.C
}

Received: 21 Jun. 2013, Revised: 26 Oct. 2013, Accepted: 27 Oct. 2013

Published online: 1 May. 2014

\begin{abstract}
This study has two phases. The first phase constructs a comprehensive model of key success factors (KSFs) for supply chain network management implementation in the semiconductor industry based on both a literature review and an expert review. The model has four dimensions: strategy, process, organization, and technology (SPOT), and $15 \mathrm{KSFs}$. The SPOT model can be a checklist for future projects. The second phase analyzed the complicated interrelationship among KSFs with Fuzzy Decision Making Trial and Evaluation Laboratory and discussed its managerial implications. Lastly, we provide two case studies to confirm the importance of the two most important factors.
\end{abstract}

Keywords: supply chain network management, key success factors, Fuzzy DEMATEL, semiconductor industry

\section{Introduction}

\subsection{Research motivation}

Supply Chain (SC) is a concept which can be considered analogous to a pipeline of physical and informational flows between suppliers and customers. From an operational point of view, this pipeline works like a process of activities that are distributed. Therefore, supply chain is not only a linear structure but actually a network [1,2]. In recent years, SC network management has become an important management approach to create closer enterprise collaborations $[3,4,5,6]$, and many companies have invested significant resources in developing information systems to obtain successful SC network management implementation. Error-free decisions regarding information technology (IT) investment for adapting a firm to its business environment are vital. However, because of the complicated nature and large scope of SC network management IT implementation, few such projects are considered successful [7,8]. In recent years, there have been increasing efforts to design an efficient information system for SC network management; however, many of these efforts have led to failure [9].

From the literature review, we found few research publications with an overview of the key success factors (KSFs) for implementing a SC network management IT system. Many publications discussed factors only from a specific perspective, such as strategy, business process, or

* Corresponding author e-mail: robertlu@mail.ntut.edu.tw, rpl@mail.tsinghua.edu.cn 
information technology; however, in the real world, overall KSFs are important for decision-making.

When enterprises implement an SC network management system, there are many factors to consider. These factors are usually with dependencies. Therefore, it is difficult for decision makers to measure a single effect from a single factor while avoiding interference from the rest of the system.

\subsection{Research objective}

The first objective was to define the comprehensive set of key success factors for implementing a SC network management IT system in the semiconductor industry. The defined model can be a critical foundation for future project managers of supply chain integration projects and ensures that all KSFs will be considered to avoid failure, which is extremely costly.

The second objective was to analyze the interrelationships of these KSFs using the Fuzzy Decision Making Trial and Evaluation Laboratory (Fuzzy DEMATEL) method and define the two KSFs that will have the greatest impact on other KSFs for SC network management implementation. To illustrate these results, real world examples were used to illustrate how the top two KSFs identified in this study affect the project implementation process. The top two factors were top management support and clear goals, objectives, and requirements.

The results of this study indicate the direction for the continuous improvements and future development of SC network management systems and can serve as a foundation for academic research in related fields.

\section{Literature Review}

\subsection{Construction of the initial KSF model}

This study summarized 78 previously published key factors and organized the factors into an initial set of KSFs for SC network management implementation. Key success factors are the most important factors that an enterprise must consider as its first priorities. If an enterprise focuses on these factors and develops appropriate strategies, it will result in successful SC network management implementation. Hence, extracting the KSFs of the SC network management implementation phase can help an enterprise efficiently allocate its resources to key areas where "things must go right".

However, there are few empirical studies concerning the KSFs of supply chain implementation with a holistic view, which serve to help companies learn about the best practice from other successful implementations. To make the results comprehensive, this study reviews the literature on the implementation of other enterprise-wide systems, such as Enterprise Resource Planning (ERP). The literature was mainly collected from journals in the fields of operation management, SC network management, and information management. To ensure a rigorous analysis, theses, dissertations, and unpublished working papers were excluded. Of the 78 factors identified, top management support and business process reengineering are mentioned in several studies. Top management support is important because it makes it easier to perform the cross organization integration task $[10,11,12,13,14,15,16]$. Business process reengineering is mentioned several times in the literature because it is critical to improve or even reengineer business processes before implementing IT solutions to support them $[10,13,17,18,14,19]$. The data accuracy and transparency in supply chain management is important because through accurate and updated information, companies can efficiently manage the product flow and information related to the issues such as production capacity, customer demand and inventory at lower costs [20,14,21,22].

Sumner [23] provided seven case studies on the implementation of enterprise-wide information management system projects. Based on these case studies, this study highlighted the issues, benefits, and critical success factors of this large-scale integrated information system. The factors evaluated in this study include management structure, the re-design of business processes, training and re-skilling, external consultants, management support, the role of the champion, discipline and standardization, and effective communication. Liu et al. [16] performed a case study of an inter-enterprise workflow-supported supply chain system and presented the key success factors of supply chain management based on experiences and lessons learned. The key factors were good cooperation and negotiation with suppliers and retailers and the use of open and standard hardware and software systems as well as standard e-business. In these two papers, top management support was referred to as an important factor.

Gunasekaran and Ngai [13] reviewed and classified previous publications regarding the application of information technology in supply chain management. There are some problems often cited in the literature when developing an IT-integrated SCM, such as a lack of integration between IT and the business model, lack of proper strategic planning, poor IT infrastructure, insufficient application of IT in virtual enterprise, and the 
inadequate implementation of IT in SCM. This study presented a framework that identifies the key areas of focus in applying information technology to supply chain management. They categorized six classifications and discussed the details in every classification. The first classification was strategic planning for IT in SCM and the details for were top management participation, a long-term business plan, developing a consortium and strategic alliances, and major changes in business processes and way the company operates. The second classification was virtual enterprise and SCM, which details discussions on partnership based on core competencies, collaborative network of firms, and training and education in IT. The third classification was e-commerce and SCM, which details discussions on education and training and government support, internet speed, logistics (including reverse), customer relationship management, security and ethics. The fourth classification was infrastructure for IT in SCM, which details discussions in IT migration, fitness for the business process, and optimal investment areas that need to be identified. The fifth classification was knowledge and IT management in SCM, which details discussions on IT training and education. The sixth classification was implementation of IT in SCM, which details discussions on a cross-functional project team with IT skills, performance measures and metrics, and a well-documented implementation plan.

Hwang et al. [17] performed research for an e-supply chain project of electronic integration in the semiconductor industry. Four critical success factors were presented to solve the challenges encountered in the project, and the four factors were business process re-engineering, business process and system integration, process and data exchange standardization, and change management.

Fawcett et al. [24] conducted a literature review, a cross-functional mail survey, and 51 in-depth case study analyses of supply chain integration and summarized the success and hindering factors for supply chain management. The factors evaluated included information transparency, collaborative planning, IT architecture /internet, formal performance tracking, adoption of a strategic SCM vision, supplier certification/reduction, target segmented customers, shared investment/benefits, accurate comprehensive measures, supplier alignment and rationization, process documentation and ownership, managerial and employee support, open information sharing, trust-based alliances, cross-trained experienced managers, supply chain education and training, and using chain advisory councils.
Langley et al. [25] discussed some major barriers and challenges that must be addressed to make supply chain technology work as intended. This study suggested that supply chain leaders must take an active role in planning, implementation, and evaluation of SCM systems.

Bose et al. [10] presented a case study of ERP and supply chain management integration in China. The factors in this paper included project management team competence, interdepartmental cooperation and communication, clear goals and objectives, vendor support, careful package selection, data analysis and conversion, dedicated resources, user training, education on new business processes, minimal customization, architecture choices, change management, internal and external integration through consolidation of system instances, use of integration technologies, standardization of data and process definitions, process flow improvement to fit with the system and business needs, addition of analytical capabilities to transform robust data access to useful business knowledge, strategies and sequences for realigning business processes, use of quality management programs, emergence of the right strategic plans to overcome power struggles and politics, software suitability, appropriate system integration strategy and technology, and information quality.

\subsection{Analysis of the interrelationships among $K S F$ s in the model}

Analysis of the interrelationships among KSFs for SC network management implementation is a multiple criteria decision-making (MCDM) problem. There are several MCDM methods, such as analytical hierarchy processes (AHP) and Decision Making Trial and Evaluation Laboratory (DEMATEL). The AHP method assumes that Key Success Factors are independent; however, decision makers often address the problem that the criteria are interdependent in the real world. For example, criterion A influences criterion $\mathrm{B}$; therefore, criterion $\mathrm{B}$ can be further improved if criterion $\mathrm{A}$ is improved. Therefore, when decision makers meet many criteria that need improvement, a better solution is to find the criteria that most strongly influence the other criteria, and the best improvement can be reached by starting with these criteria.

The DEMATEL method, which was developed by the Science and Human Affairs Program of the Battelle Memorial Institute of Geneva between 1972 and 1976, was used to study and solve a cluster of intertwined problems and contribute to the identification of workable 
solutions using a hierarchical structure [26,27,28]. It is an appropriate method that helps in gathering group knowledge, visualizing the causal relationships of the criteria, and indicates the degree of influence through a cause-effect diagram. DEMATEL is commonly used in various types of MCDM problems. Lin and Tzeng [29] proposed developing strategies and operation models using DEMATEL for the administration of science park to advance the value of the park. DEMATEL can convert the relationships between criteria into a visual structural model and can be used as a way to handle the inner dependencies within a set of criteria.

\section{Phase 1 - constructing the SPOT model}

There were two phases in this study. The first phase used a literature review to construct an initial KSF model, and the model was reviewed by experts who have experience in SC network management system implementation to finalize the SPOT model. The second phase consisted of using Fuzzy DEMATEL to calculate the interrelationships between each key success factor.

\subsection{Expert Review}

From the literature review, we found 78 previously published key factors and organized the factors into an initial set of KSFs for SC network management system implementation. This information was used as the foundation for the ensuing expert review.

To combine academic knowledge with practical experience in order to finalize a comprehensive KSF model, this study used the initial set of KSFs as a foundation, and a series of expert review discussions were conducted to obtain a diverse array of valuable experiences and perspectives on the topic "Identifying key success factors for SC network management implementation in semiconductor industry".

The participants of these expert review discussions were divided in two groups; the first group included individuals from an academic setting, and the second group included individuals from industry. The academic group was divided into two fields: "industrial engineering and management," which consisted of five participants, and "technology management," which consisted of two participants. The industry group was divided into two fields: "information technology," which consisted of four participants, and "business," which consisted of two participants. In expert review, the total number of participants was 13 .
Five expert review discussions were conducted, and each group discussion was held with four to six participants. The participants were selected to comply with the guidelines of homogeneity, heterogeneity and representation proposed by Morgan [30] to form an appropriate expert review meeting. All participants were familiar with SC network management and had different backgrounds and expertise. The participants recruited from industry all participated in a successful e-supply chain project between the Taiwan Semiconductor Manufacturing Company (TSMC), which is the largest semiconductor foundry in the world, and Advanced Semiconductor Engineering Inc. (ASE), which is the worlds largest semiconductor assembly, testing, and packaging service provider.

All expert review discussions were moderated by a same moderator. The moderator had 18 years of industrial experience with SC network management system, had full understanding of the topic and purpose of the expert review discussions, and had participated in the aforementioned e-supply chain project. The following five questions were asked in each session to ensure the group discussion stayed focused on the topic:

-Which KSFs do you think are appropriate for SC network management system implementation in the semiconductor industry?

-Which dimensions do you think are appropriate for SC network management system implementation in the semiconductor industry?

-Which KSFs and dimensions can be consolidated?

-Which KSFs should be put under which dimension?

-Is the KSF model for SC network management implementation a comprehensive model?

Each expert review interview lasted approximately 60 to 90 minutes, and the data were recorded by note taking. The moderator made a summary note after each session to facilitate data analysis. In each discussion, the moderator and participants followed the above five questions to lead the discussion. The four dimensions discussed were strategy, process, organization, and technology. In the discussion, the panelists deleted or combined the initial factors to obtain the SPOT model. 
Table 1 The SPOT model

\begin{tabular}{ll}
\hline Dimension & Factor \\
& $\begin{array}{l}\text { F1. Top management support } \\
\text { F2. Clear goals, objectives, and business } \\
\text { requirements }\end{array}$ \\
& $\begin{array}{c}\text { F3. An appropriate SC network management } \\
\text { system implementation strategy }\end{array}$ \\
& F4. Compliance of related policies and regulations \\
& $\begin{array}{l}\text { F5. Business process re-engineering } \\
\text { F6. The integration of business process and } \\
\text { information systems }\end{array}$ \\
& F7. Processes designed to ensure interdepartmental \\
& cooperation
\end{tabular}

\subsection{The SPOT model}

The result of data analysis was a KSF model for SC network management implementation in the semiconductor industry and is shown in Table 1. This KSF model was named the "SPOT model" because it consisted of four dimensions (strategy, process, organization, and technology) and 15 key success factors.

\section{Phase 2 - Analyze the SPOT model using Fuzzy DEMATEL}

\subsection{Fuzzy DEMATEL}

\subsubsection{DEMATEL method}

The DEMATEL method was developed by the Geneva Research Centre of the Battelle Memorial Institute, which was developed to study the structural relationships in a complicated cluster of problems. The DEMATEL method can be used to indicate the degree of influence between factors and visualize the causal relationship among the factors through a cause-effect diagram. The method uses directed graphs to demonstrate the directed relationship among these factors. Suppose there is a set of $\mathrm{n}$ factors in the decision-making problem, $F=\{F 1, F 2, \ldots, F N\}$. The essential definitions are described below:
Definition 1: The pair-wise comparison scale has five levels, which are "No influence (0)", "Low influence (1)", "Medium influence (2)", "High influence (3)", and "Very high influence (4)" In addition, "(\#)" indicates the score of this level.

Definition 2: The initial direct relationship matrix $X$ is an $n \times n$ matrix, which is obtained by a pairwise comparison, $X=\left[x_{i j}\right]_{n \times n}$. The element $x_{i j}$ denotes that the degree criteria $i$ influences criteria $j$.

Definition 3: The normalized direct relationship matrix $D, D=\left[d_{i j}\right]_{n \times n}$. Using formula (3.1) and (3.2), $0 \leq d_{i j} \leq 1$ can be obtained, and the diagonal elements are 0 .

$$
\begin{gathered}
s=\frac{1}{\max _{1 \leq i \leq n} \sum_{j=1}^{n} x_{i j}}, i, j=1,2, \ldots, n \\
D=s \cdot X
\end{gathered}
$$

Definition 4:The total relationship matrix $T$, $T=\left[t_{i j}\right]_{n \times n} . T$ is calculated using formula (3.3), and $I$ denotes the identity matrix.

$$
T=D(I-D)^{-1}
$$

Definition 5: $R$ and $C$ denote the sum of the row and the sum of the column within matrix $T$, respectively. $R$ and $C$ can be acquired using formulas (3.4) and (3.5), respectively.

$$
\begin{aligned}
& R_{i}=\sum_{j=1}^{n} t_{i j} \\
& C_{j}=\sum_{i=1}^{n} t_{i j}
\end{aligned}
$$

$R_{i}$ is the sum of the row and shows the total given effects, directly and indirectly. $C_{j}$ is the sum of the column and shows the total received effects, directly and indirectly. Hence, when $i=j$, the sum $\left(R_{i}+C_{i}\right)$ denotes the total effects given and received by factor $i$ and indicates the importance of factor $i$ in the decision-making problem. In addition, the difference $\left(R_{i}-C_{i}\right)$ denotes the net effect by factor $i$ and can be used to classify factor $i$ into the cause group or the effect group.

Definition 6: A cause-effect diagram is drawn with the horizontal axis $(R+C)$ and vertical axis $(R-C)$. Each factor can be mapped by the dataset $(R+C, R-C)$.

\subsubsection{The Fuzzy DEMATEL}

Fuzzy logic is a mathematical way to represent and handle vagueness in decision-making, and it is beneficial to convert linguistic terms into fuzzy numbers. The 
concept of linguistic variables is extremely useful in dealing with decision-making situations, which are too complex or ill-defined to be reasonably described using conventional quantitative expressions. A linguistic variable is a variable with lingual expression as its value. For example, "No influence," "Medium influence," and "High influence" refer to the natural representation of the preference or judgment. The essential definitions of fuzzy logic are described as the following:

Definition 7: A fuzzy set $\widetilde{K}$ is a subset of a universe of discourse $X$ and is characterized by a membership function $\mu_{\widetilde{K}}(x)$ representing a mapping $\mu_{\widetilde{K}}: X \rightarrow[0,1] . X=\{x\}$ represents a collection of element $x$, and the function value of $\mu_{\widetilde{K}}(x)$ for the fuzzy set $\widetilde{K}$ is called the membership value of $x$ in $\widetilde{K}$, which represents the degree of truth that $x$ is an element of the fuzzy set $\widetilde{K}$. It is assumed that $\mu_{\widetilde{K}}(x) \in[0,1]$, where $\mu_{\widetilde{K}}(x)=1$ reveals that $x$ belongs to $\widetilde{K}$ completely, whereas $\mu_{\widetilde{K}}(x)=0$ indicates that $x$ does not belong to the fuzzy set $\widetilde{K}$.

Definition 8: A fuzzy number $\widetilde{N}$ is a fuzzy subset in the universe of discourse $X$.

Definition 9: A triangular fuzzy number $\widetilde{N}$ can be defined as a triplet $(l, m, r)$, and the membership function $\mu_{\widetilde{K}}(x)$ is defined as:

$$
\mu_{\widetilde{N}}(x)=f(x)=\left\{\begin{array}{rr}
0, & x<1 \\
(x-1) /(m-1), & l \leq x \leq m \\
(r-x) /(r-m), & m \leq x \leq r \\
0, & x>r
\end{array}\right.
$$

Where $l, m$, and $r$ are real numbers and $l \leq m \leq r$.

Definition 10: Calculate the average TFN score. The average matrix $A$ is obtained by averaging all decision makers scores, $A=\left[a_{i j}\right]_{n \times n}$ and is calculated using formula (3.7).

$$
a_{i j}=\left(L_{i j}, M_{i j}, R_{i j}\right)=\frac{1}{H} \sum_{k=1}^{H} \widetilde{x}_{i j}^{k}=\frac{1}{H} \sum_{k=1}^{H}\left(l_{i j}, m_{i j}, r_{i j}\right)
$$

Where $H$ indicates the numbers of decision makers to be asked, and $\widetilde{x}_{i j}^{k}$ is a TFN score denotes the degree that factor $i$ affects factor given by the $k_{t h}$ decision maker.

Definition 11: Calculate the BNP value. The BNP value of a TFN can be calculated using formula (3.8).

$$
B N P_{i j}=L_{i j}+\frac{\left(R_{i j}-L_{i j}\right)+\left(M_{i j}-L_{i j}\right)}{3}
$$

After deffizification, an average crisp value matrix will be obtained, and this value can be used in the rest of the DEMATEL method.
Table 2 Linguistic variable table

\begin{tabular}{lc}
\hline Value of linguistic variable & TFN \\
\hline Very high influence & $(3,4,4)$ \\
High influence & $(2,3,4)$ \\
Medium influence & $(1,2,3)$ \\
Low influence & $(0,1,2)$ \\
No influence & $(0,0,1)$ \\
\hline
\end{tabular}

The procedure of the Fuzzy DEMATEL method is explained below.

Step 1: Identifying the goal for decision-making and extracting the evaluation criteria. In decision-making problems, the first step must be identifying the decision goal. Second, it is necessary to develop a set of key criteria for evaluation; for example, forming a committee is an effective way to gather group knowledge to extract the evaluation criteria of the goal and design the questionnaire using the set of criteria.

Step 2: Acquiring and aggregating the opinions of the decision makers. This step generates a survey to obtain the opinions of the decision makers. For dealing with the ambiguities of human thought, fuzzy set theory is incorporated with the DEMATEL method. The linguistic variables scale and corresponding TFN are presented in Table 2. Decision makers assign the linguistic variables to the pairwise comparison between criteria, and these linguistic variables will be converted into TFN. Using the Centroid method, these fuzzy assessments are aggregated and defuzzified as a crisp value through definitions 10 and 11. After deffizification, an average direct relationship matrix will be obtained, and the matrix can be used in the rest of the DEMATEL method.

Step 3: Computing the total relationship matrix. Based on the average direct relationship matrix, the normalized direct relationship matrix can be obtained through definition 3, and the total relationship matrix can be acquired using definition 4 .

Step 4: Obtaining and analyzing the cause-effect diagram. According to definition 5, the sum of row " $R_{i}$ " and the sum of column " $C_{i}$ " can be obtained, and the dataset for each factor can be calculated. By definition 6, the cause-effect diagram can be drawn with the horizontal axis " $R+C$ " and the vertical axis " $R-C$ ". Hence, the cause-effect diagram can be used to illustrate the complicated interrelationship of factors. Finally, the cause-effect diagram can be analyzed, and its managerial implications can be interpreted by decision makers. 
Table 3 The job level and function of surveyed respondents

\begin{tabular}{ccccc}
\hline Department & $\begin{array}{c}\text { Director } \\
\text { \& Senior } \\
\text { manager }\end{array}$ & Manager & $\begin{array}{c}\text { Senior } \\
\text { engineer }\end{array}$ & Sub-total \\
\hline IT & 2 & 6 & 2 & 10 \\
Business & 5 & 2 & 0 & 7 \\
Sub-total & 7 & 8 & 2 & 17 \\
\hline
\end{tabular}

\subsection{The questionnaire survey}

A questionnaire was designed based on the SPOT model. This study surveyed the 17 managers and executives that participated in the e-supply chain project between TSMC and ASE. The surveyed respondents were carefully selected to obtain the experiences and opinions from different job levels and functions (shown in Table 3).

\subsection{Interrelationships among the 15 factors evaluated}

After the questionnaire survey, Fuzzy DEMATEL was employed to capture the complicated interrelationships between the 15 factors evaluated. First, each survey result was converted into a fuzzy matrix with a linguistic variable table.

Formula (7) was used to average the assessment data of the respondents, and formula (8) was used to defuzzify the averaged fuzzy matrix into an initial direct relationship matrix. Based on the initial direct relationship matrix, the normalized direct relationship matrix was obtained using formula (1)-(2). Finally, the total relationship matrix was acquired using formula (3). Table 4 shows the total relationship matrix.

For each factor $i$, the sum of row " $R_{i}$ ", the sum of column " $C_{i}$ ", $\left(R_{i}+C_{i}\right)$ and $\left(R_{i}-C_{i}\right)$ were calculated using formulae (4)-(5) (shown in Table 5). " $R_{i}$ " indicates the degree that factor $i$ influences other factors, whereas " $C_{i}$ " indicates the degree that factor $i$ is influenced by other factors. Therefore, denotes the total effects given and received by factor $i$ and indicates the degree of importance of factor $i$ in the entire system. Furthermore, $\left(R_{i}-C_{i}\right)$ represents the net effect that factor $i$ has on the system and can be used to classify factor $i$ into the cause group or the effect group. Factor $i$ belongs to the cause group when $\left(R_{i}-C_{i}\right)$ is positive; otherwise, it belongs to the effect group.

The cause-effect diagram is presented in Figure 1 by mapping the dataset $\left(R_{i}+C_{i}, R_{i}-C_{i}\right)$ with the horizontal axis-total effect " $R+C$ " and the vertical axis-net effect
" $R-C$ ".

Table 5 The sum of the effects given and received for each factor

\begin{tabular}{ccccc}
\hline Code & $\mathrm{R}$ & $\mathrm{C}$ & $\begin{array}{c}\text { Total effect } \\
(\mathrm{R}+\mathrm{C})\end{array}$ & $\begin{array}{c}\text { Net effect } \\
(\mathrm{R}-\mathrm{C})\end{array}$ \\
\hline F1 & 2.4711 & 1.144 & 3.855 & 1.567 \\
F2 & 2.593 & 1.300 & 3.893 & 1.293 \\
F3 & 1.999 & 1.659 & 3.659 & 0.340 \\
F4 & 1.608 & 1.007 & 2.615 & 0.601 \\
F5 & 2.044 & 1.981 & 4.025 & 0.063 \\
F6 & 1.625 & 2.052 & 3.677 & -0.428 \\
F7 & 1.584 & 1.854 & 3.439 & -0.270 \\
F8 & 1.792 & 1.637 & 3.429 & 0.156 \\
F9 & 1.462 & 2.188 & 3.650 & -0.726 \\
F10 & 1.517 & 1.994 & 3.510 & -0.477 \\
F11 & 1.467 & 1.719 & 3.186 & -0.253 \\
F12 & 1.428 & 1.761 & 3.189 & -0.333 \\
F13 & 1.319 & 1.892 & 3.211 & -0.573 \\
F14 & 1.302 & 1.938 & 3.241 & -0.636 \\
F15 & 1.283 & 1.607 & 2.890 & -0.324 \\
\hline
\end{tabular}

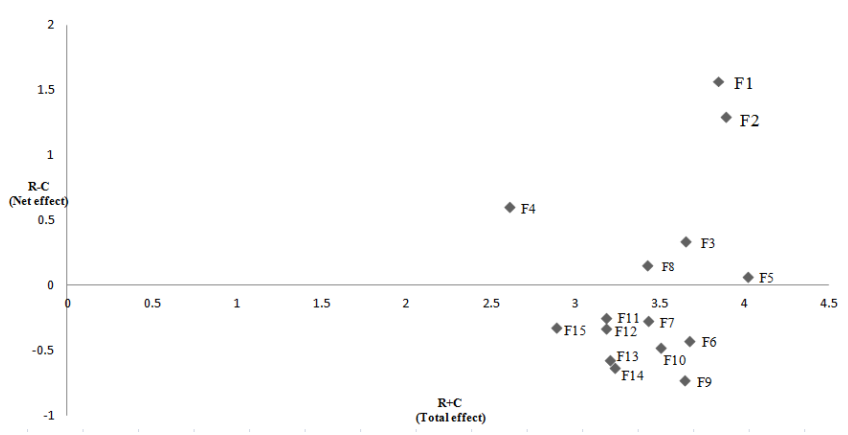

Fig. 1 The cause-effect diagram

\section{Resutls and discussion}

\subsection{Results}

By analyzing the cause-effect diagram, some valuable information can be obtained for future project managers to make decisions. The managerial implications of this analytical result are summarized as follows. 


\begin{tabular}{|c|c|c|c|c|c|c|c|c|c|c|c|c|c|c|c|}
\hline F1 & 0.089 & 0.171 & 0.190 & 0.136 & 0.222 & 0.195 & 0.201 & 0.196 & 0.240 & 0.211 & 0.184 & 0.185 & 0.172 & 0.169 & 0.147 \\
\hline F2 & 0.141 & 0.095 & 0.192 & 0.121 & 0.217 & 0.212 & 0.189 & 0.152 & 0.212 & 0.193 & 0.177 & 0.182 & 0.171 & 0.184 & 0.154 \\
\hline F3 & 0.087 & 0.103 & 0.091 & 0.082 & 0.153 & 0.165 & 0.143 & 0.134 & 0.177 & 0.163 & 0.128 & 0.154 & 0.145 & 0.143 & 0.130 \\
\hline F4 & 0.093 & 0.106 & 0.115 & 0.052 & 0.134 & 0.115 & 0.116 & 0.098 & 0.138 & 0.117 & 0.112 & 0.117 & 0.103 & 0.106 & 0.088 \\
\hline F5 & 0.091 & 0.101 & 0.126 & 0.078 & 0.110 & 0.179 & 0.166 & 0.129 & 0.188 & 0.169 & 0.137 & 0.135 & 0.150 & 0.163 & 0.122 \\
\hline F6 & 0.065 & 0.080 & 0.094 & 0.054 & 0.125 & 0.094 & 0.114 & 0.104 & 0.140 & 0.126 & 0.110 & 0.113 & 0.141 & 0.141 & 0.124 \\
\hline F7 & 0.067 & 0.077 & 0.100 & 0.058 & 0.123 & 0.130 & 0.083 & 0.107 & 0.141 & 0.149 & 0.114 & 0.111 & 0.113 & 0.117 & 0.095 \\
\hline F8 & 0.082 & 0.093 & 0.113 & 0.060 & 0.142 & 0.145 & 0.138 & 0.083 & 0.156 & 0.154 & 0.123 & 0.117 & 0.141 & 0.137 & 0.109 \\
\hline F9 & 0.073 & 0.072 & 0.094 & 0.056 & 0.122 & 0.120 & 0.113 & 0.091 & 0.090 & 0.116 & 0.102 & 0.099 & 0.111 & 0.113 & 0.090 \\
\hline F10 & 0.065 & 0.075 & 0.092 & 0.055 & 0.127 & 0.117 & 0.123 & 0.105 & 0.144 & 0.086 & 0.126 & 0.096 & 0.111 & 0.113 & 0.084 \\
\hline F11 & 0.066 & 0.077 & 0.098 & 0.056 & 0.122 & 0.109 & 0.115 & 0.106 & 0.130 & 0.124 & 0.073 & 0.100 & 0.100 & 0.104 & 0.088 \\
\hline F12 & 0.066 & 0.075 & 0.099 & 0.058 & 0.111 & 0.111 & 0.094 & 0.091 & 0.114 & 0.107 & 0.094 & 0.073 & 0.114 & 0.122 & 0.100 \\
\hline F13 & 0.056 & 0.061 & 0.086 & 0.050 & 0.094 & 0.124 & 0.090 & 0.084 & 0.108 & 0.099 & 0.081 & 0.087 & 0.074 & 0.121 & 0.104 \\
\hline F14 & 0.053 & 0.056 & 0.081 & 0.049 & 0.094 & 0.114 & 0.089 & 0.080 & 0.105 & 0.095 & 0.081 & 0.098 & 0.126 & 0.075 & 0.109 \\
\hline F15 & 0.051 & 0.058 & 0.088 & 0.043 & 0.086 & 0.122 & 0.081 & 0.077 & 0.106 & 0.085 & 0.076 & 0.096 & 0.120 & 0.130 & 0.064 \\
\hline
\end{tabular}

First, through the cause-effect diagram, these key success factors can be divided into two groups. If the " $R-C$ " (net effect) value of factor $i$ is positive, it belongs to the cause group; otherwise, it belongs to effect group. The cause group includes F1, F2, F3, F4, F5 and F8, whereas the effect group includes F6, F7, F9, F10, F11, F12, F13, F14 and F15. The effect group factors are easily affected by the cause group factors. Hence, if companies want to obtain high performance in terms of the effect group factors, they should control and improve the cause group factors in advance.

Second, the top two factors with the highest " $R-C$ " values are top management support (F1) and clear goals, objectives, and business requirements (F2). These two factors have the most influence $(R-C)$ on other factors; at the same time, these two factors have the second and third highest intensity relationships $(R+C)$. Hence, they are the most important factors and should be focused on first. In addition, to Compliance of related policies and regulations $(\mathrm{F} 4)$ has the third highest " $R-C$ " value; however, its " $R+C$ " (total effect) value is the lowest. This indicates that this factor affects other factors and is not easily affected by other factors. However, change management (F9), which has a strong negative $R-C$ value, is easily affected by other factors.

Third, business process re - engineering (F5), cleargoals, objectives, and requirements (F2), and top management support $(\mathrm{F} 1)$ are the factors that have the highest $R+C$ value. These results indicate that these three factors have the highest intensity of relation to other factors and have a large effect on the system.

Fourth, the factors that have the top four $R-C$ values are all in the dimension "strategy". Therefore, strategy is the most influential dimension. The factors in this dimension should be carefully considered because this dimension affects many other factors.

\subsection{Discussion}

The top two factors that have the highest $R-C$ values are top management support and clear goals, objectives, and business requirements. We want to analyze a successful experience in SC network management system implementation and we want to confirm and illustrate how these two factors will affect the successful implementation of an SC network management system.

\subsubsection{Top management support}

As key supply chain activities in a company usually exist across multiple organizations and the financial resource of implementing a SC network management system is high, top management support to form a cross-organizational project team and allocate sufficient financial resources for achieving the projects success is imperative [32]. The e-SCM project was led by the senior vice president of IT with strong steering committee members that consisted of senior managers from Sales and Marketing, Manufacturing Operation, and Corporate Planning at TSMC and ASE. The active participation of the top management set a clear vision and provided valuable advice at critical points that profoundly affected the project results. In the emergence of the e-SCM project, top management sketched the concept of "Virtual Fab" and defined the high-level project implementation roadmap for the entire e-SCM project team to follow. The steering committee had been active in leading the whole project and holding review meetings on a regular basis. As the top management realized that the benefits of e-SCM would exponentially increase only if the number of connected companies was maximized, one of the most critical decisions made by the committee was to urge the internationalization of the data transfer protocol developed by TSMC and ASE via RosettaNet, which is a globally 
supported standards development organization for collaborative commerce. The data standards this organization defines are widely adopted by companies to support inter-company business processes for efficient SC network management. Therefore, implementation of this protocol significantly accelerated the e-SCM solution that is being widely adopted by other companies in the semiconductor industry supply chain.

\subsubsection{Clear goals, objectives, and business requirement}

The goal of the e-SCM project, which was to integrate key operational activities and data between TSMC and ASE and extend the information and transaction interface to their joint customers, had been very clear since the project kickoff. Under the guidance of the stated goals, the specific objectives that the project intended to reach in the six-year of the duration of the project were defined accordingly: (1) to streamline and integrate key business processes, (2) to improve the transparency of information exchange, and (3) to increase the speed of information delivery. All of the project activities, including business requirement collection, change management plan development, and IT system design, were aligned to the projects goals and objectives.

To fulfill the needs of all business transactions among TSMC, ASE and their joint customers, the management team needed to ensure that the system solution was designed as comprehensively and universally as possible without sacrificing flexibility. One good example that illustrates the incorporation of commonality and customization of the e-SCM system is that, as shown in Figure 2, the standard e-PO was implemented for normal procedures, whereas a customized Purchase Order (PO) process was designed to satisfy specific requirements: one international company with multiple worldwide regional offices requested a special business process. In this process, when a master PO was sent to TSMC, the master PO could be split into multiple sub-POs for each of its regional offices for shipping and invoicing purposes.

\section{Conclusions}

This study proposes a KSF model for SC network management system implementation. The SPOT model was initially constructed based on a literature review and was finalized through expert review to obtain valuable industrial experience. This KSF model can be used as a checklist to prevent companies from overlooking any

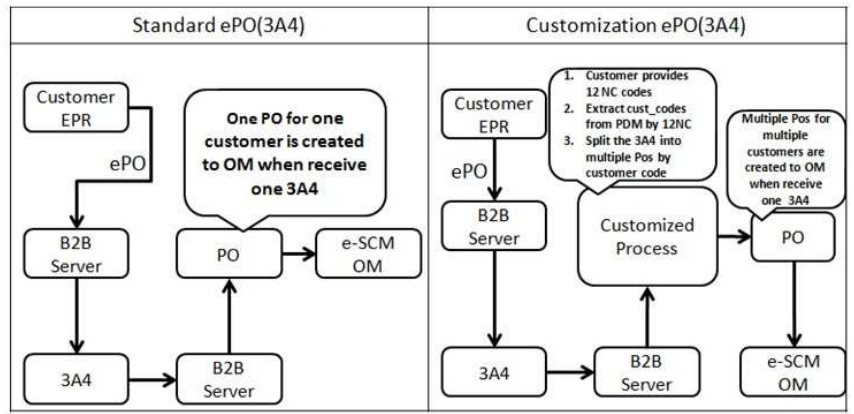

PO: Purchase Order;

OM: Order Management;

3A4: The Partner Interface Processes (PIP) number for e-Order defined by RosettaNet

Fig. 2 e-SCM process design for standard and customization

important factors that may result in the failure of implementation of an SC network management system project. This study adopted the Fuzzy DEMATEL method to analyze and illustrate the complicated interrelationships among KSFs. Through the analysis process, the KSFs can be categorized to support future project managers in making important decisions. Particularly, top management support and clear goals, objectives, and business requirements play an important role in SC network management system implementation projects. These two factors have high intensity relationships and are the most influential factors. Therefore, companies should consider these two factors first. Furthermore, all factors in the "strategy" dimension should be carefully considered because these factors easily affect other factors. The results of this study will be valuable for companies when allocating limited resources in SC network management system implementation projects.

\section{Acknowledgement}

This research is partially supported by the National Science Council, Taiwan, project number NSC98-2218-E-027-007. The authors are grateful to the anonymous referee for a careful checking of the details and for helpful comments that improved this paper.

\section{References}

[1] H. J. Ahn, H. Lee and S. J. Park, A flexible agent system for change adaptation in supply chains. Expert Systems with Applications, 25, 603-618 (2003). 
[2] V. Bhaskar and P. Lallement, Activity routing in a distributed supply chain: Performance evaluation with two inputs. Journal of Network and Computer Applications, 31, 402-428 (2008).

[3] B. Ageron, A. Gunasekaran and A. Spalanzani, Sustainable supply management: An empirical study. International Journal of Production Economics, 140, 168-182 (2012).

[4] M. Ben-Daya, R. Asad and M. Seliaman, An integrated production inventory model with raw material replenishment considerations in a three layer supply chain. International Journal of Production Economics (2010), In press.

[5] A. Kinra and H. Kotzab, A macro-institutional perspective on supply chain environmental complexity. International Journal of Production Economics, 115, 283-295 (2008).

[6] Q. Su, J.-H. Shi and S.-J. Lai, Study on supply chain management of Chinese firms from the institutional view. International Journal of Production Economics, 115, 362-373 (2008).

[7] S. Li, S. S. Rao, T. S. Ragu-Nathan and B. Ragu-Nathan, Development and validation of a measurement instrument for studying supply chain management practices. Journal of Operations Management, 23, 618-641 (2005).

[8] I.-L. Wu, and C.-H. Chuang, Analyzing contextual antecedents for the stage-based diffusion of electronic supply chain management. Electronic Commerce Research and Applications, 8, 302-314 (2009).

[9] M. J. Tarokh and J. Soroor, Supply Chain Management Information Systems Critical Failure Factors, Service Operations and Logistics, and Informatics, 2006. SOLI '06. IEEE International Conference on (2006), 425-431.

[10] I. Bose, R. Pal and A. Ye, ERP and SCM systems integration: The case of a valve manufacturer in China. Information and Management, 45, 233-241 (2008).

[11] R. Bose and X. Luo, Integrative framework for assessing firms potential to undertake Green IT initiatives via virtualization - A theoretical perspective. The Journal of Strategic Information Systems, 20, 38-54 (2011).

[12] A. W. Gatian, R. M. Brown and J. O. Hicks Jr, Organizational innovativeness, competitive strategy and investment success. The Journal of Strategic Information Systems, 4, 43-59 (1995).

[13] A. Gunasekaran and E. W. T. Ngai, Information systems in supply chain integration and management. European Journal of Operational Research, 159, 269-295 (2004).

[14] S.C.L. Koh, A. Gunasekaran and T. Goodman, Drivers, barriers and critical success factors for ERPII implementation in supply chains: A critical analysis. The Journal of Strategic Information Systems, 20, 385-402 (2011).

[15] W. Li, P. K. Humphreys, A. C. L. Yeung and T. C. E. Cheng, The impact of supplier development on buyer competitive advantage: A path analytic model. International Journal of Production Economics, 135, 353-366 (2012).

[16] J. Liu, S. Zhang and J. Hu, A case study of an interenterprise workflow-supported supply chain management system. Information and Management, 42, 441-454 (2005).
[17] B.-N. Hwang, S.-C. Chang, H.-C. Yu and C.-W. Chang, Pioneering e-supply chain integration in semiconductor industry: a case study. The International Journal of Advanced Manufacturing Technology, 36, 825-832 (2008).

[18] T. Kobayashi, M. Tamaki and N. Komoda, Business process integration as a solution to the implementation of supply chain management systems. Information and Management, 40, 769780 (2003).

[19] Y. Merali, T. Papadopoulos and T. Nadkarni, Information systems strategy: Past, present, future? The Journal of Strategic Information Systems, 21, 125-153 (2012).

[20] P. Hanafizadeh and M. H. Sherkat, Designing fuzzy-genetic learner model based on multi-agent systems in supply chain management. Expert Systems with Applications, 36, 1012010134 (2009).

[21] E. W. T. Ngai, D. C. K. Chau and T. L. A. Chan, Information technology, operational, and management competencies for supply chain agility: Findings from case studies. The Journal of Strategic Information Systems, 20, 232-249 (2011).

[22] M.-M. Yu, S.-C. Ting and M.-C. Chen, Evaluating the crossefficiency of information sharing in supply chains. Expert Systems with Applications, 37, 2891-2897 (2010).

[23] M. Sumner, Critical success factors in enterprise wide information management systems projects, Proceedings of the 1999 ACM SIGCPR conference on Computer personnel research (1999). ACM, New Orleans, Louisiana, United States, pp. 297-303.

[24] S. E. Fawcett, G. M. Magnan and M. W. McCarter, Benefits, barriers, and bridges to effective supply chain management. Supply Chain Management: An International Journal, 13, 3548 (2008).

[25] C. J. Langley, Managing Supply Chains: A Logistic Approach with Student CD. South-Western, (2008).

[26] E. Fontela and A. Gabus, DEMATEL, innovative methods, Report no. 2 structural analysis of the world problematique. Battelle Geneva Research Institute, (1974).

[27] A. Gabus and E. Fontela, World problems an invitation to further thought within the framework of DEMATEL. Geneva: Battelle Geneva Research Centre, Switzerland, (1972).

[28] A. Gabus and E. Fontela, Perceptions of the world problematique: Communication procedure, communicating with those bearing collective responsibility, DEMATEL report no. 1. Geneva: Battelle Geneva Research Centre, Switzerland, (1973).

[29] C.-L. Lin and G.-H. Tzeng, A value-created system of science (technology) park by using DEMATEL. Expert Systems with Applications, 36, 9683-9697 (2009).

[30] D. L. Morgan, Focus groups as qualitative research. Sage Publications, (1988).

[31] J. Motwani, M. Madan and A. Gunasekaran, Information technology in managing global supply chains. Logistics Information Management, 13, 320-327 (2000).

[32] R. G. R. Jr, H. Chen, R. Upreti, S. E. Fawcett and F.G. Adams, The moderating role of barriers on the relationship 
between drivers to supply chain integration and firm performance. International Journal of Physical Distribution \& Logistics Management, 39, 826-840 (2009).

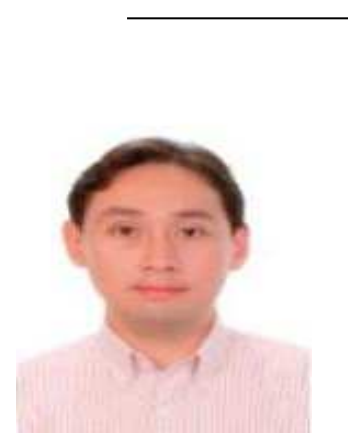

Ta-Ping Lu is an Assistant Professor of Department of Industrial Engineering and Management at the National Taipei University of Technology, Taiwan. He earned his Ph.D. from Purdue University. Dr. Lu was formerly a project manager with Taiwan Semiconductor Manufacturing Company. Entering academia after thirteen years of technical and managerial experience in supply chain management and enterprise information systems, Dr. Lus current research interests are in supply chain management, healthcare management, and management information system.

Pei-Luen Patrick Rau is

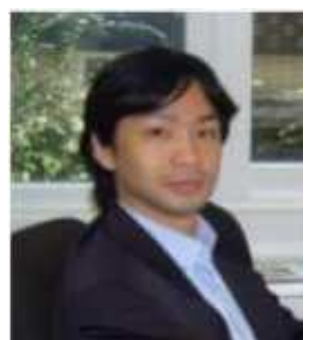
a professor in the department of Industrial Engineering at Tsinghua University in Beijing. Since 2002, he has founded and directs HCI and Usability research center at Tsinghua University, and also directs Institute of Human Factors and Ergonomics at Tsinghua University. He was an adjunct professor at Yonsei University in Korea, a visiting scholar at Microsoft Research Asia in China, visiting professor at the RWTH Aachen University in Germany, and a visiting professor at the Chuo University in Japan. His research themes include cross-cultural design, technology acceptance, and design for elderly. Prof. Rau has published several articles on these topics in the $\mathrm{HCI}$ and related journals and conferences.

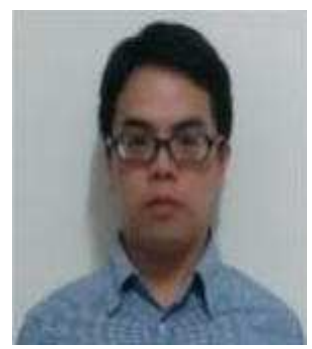

Tang-Zuo Liou earned his masters degree from Department of Industrial Engineering and Management, National Taipei University of Technology by 2010. His current research interests are in supply chain management, decision analysis, and experiment design.

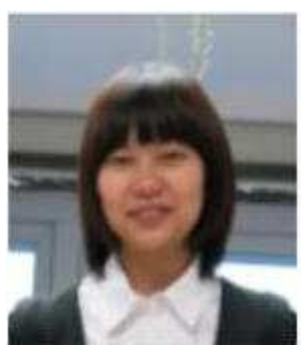

Yi-Hsin Yang earned her masters degree from Department of Industrial Engineering and Management, National Taipei University of Technology by 2013. Her current research interests are in supply chain management, decision analysis, and experiment design. 